\title{
Magnitude of Potentially Inappropriate Thrombophilia Testing in the Inpatient Hospital Setting
}

\author{
Eric Mou, MD, Henry Kwang, MD¹, Jason Hom, MD¹, Lisa Shieh, MD¹, Andre Kumar, MD, \\ llana Richman, $\mathrm{MD}^{1}$, Caroline Berube, $\mathrm{MD}^{2}$
}

${ }^{1}$ Department of Medicine, Stanford University, Stanford, California; '2Division of Hematology, Stanford University, Stanford, California.

\begin{abstract}
Laboratory costs of thrombophilia testing exceed an estimated $\$ 650$ million (in US dollars) annually. Quantifying the prevalence and financial impact of potentially inappropriate testing in the inpatient hospital setting represents an integral component of the effort to reduce healthcare expenditures. We conducted a retrospective analysis of our electronic medical record to evaluate 2 years' worth of inpatient thrombophilia testing measured against preformulated appropriateness criteria. Cost data were obtained from the Centers for Medicare and Medicaid Services 2016 Clinical Laboratory Fee Schedule. Of
\end{abstract}

the 1817 orders analyzed, 777 (42.7\%) were potentially inappropriate, with an associated cost of $\$ 40,422$. The tests most frequently inappropriately ordered were Factor $V$ Leiden, prothrombin gene mutation, protein $\mathrm{C}$ and $\mathrm{S}$ activity levels, antithrombin activity levels, and the lupus anticoagulant. Potentially inappropriate thrombophilia testing is common and costly. These data demonstrate a need for institution-wide changes in order to reduce unnecessary expenditures and improve patient care. Journal of Hospital Medicine. 2017;12:735-738. (C) 2017 Society of Hospital Medicine
Venous thromboembolism (VTE) affects more than 1 million patients and costs the US healthcare system more than $\$ 1.5$ billion annually. ${ }^{1}$ Inherited and acquired thrombophilias have been perceived as important risk factors in assessing the risk of VTE recurrence and guiding the duration of anticoagulation.

Thrombophilias increase the risk of a first thrombotic event, but existing data have failed to demonstrate the usefulness of routine thrombophilia screening on subsequent management. ${ }^{2,3}$ Moreover, thrombophilia testing ordered in the context of an inpatient hospitalization is limited by confounding factors, especially during an acute thrombotic event or in the setting of concurrent anticoagulation. ${ }^{4}$

Recognizing the costliness of routine thrombophilia testing, The American Society of Hematology introduced its Choosing Wisely campaign in 2013 in an effort to reduce test ordering in the setting of provoked VTEs with a major transient risk factor. ${ }^{5}$ In order to define current practice behavior at our institution, we conducted a retrospective study to determine the magnitude and financial impact of potentially inappropriate thrombophilia testing in the inpatient setting.

\section{METHODS}

We performed a retrospective analysis of thrombophilia testing across all inpatient services at a large, quaternary-care academic institution over a 2 -year period. Electronic medical record data containing all thrombophilia tests ordered on

\footnotetext{
*Address for correspondence and reprint requests: Eric Mou, MD, 300 Pasteur Drive, Stanford, CA 94304; Telephone: 515-771-9639; Fax: 650-725-8381; E-mail: emou@stanford.edu

Additional Supporting Information may be found in the online version of this article.

Received: January 20, 2017; Revised: April 21, 2017;

Accepted: April 28, 2017
}

2017 Society of Hospital Medicine DOI 10.12788/jhm.2819 inpatients from June 2013 to June 2015 were obtained. This study was exempt from institutional review board approval.

Inclusion criteria included any inpatient for which thrombophilia testing occurred. Patients were excluded if testing was ordered in the absence of VTE or arterial thrombosis or if it was ordered as part of a work-up for another medical condition (see Supplementary Material).

Thrombophilia testing was defined as any of the following: inherited thrombophilias (Factor V Leiden or prothrombin 20210 gene mutations, antithrombin, or protein $\mathrm{C}$ or $\mathrm{S}$ activity levels) or acquired thrombophilias (lupus anticoagulant [Testing refers to the activated partial thromboplastin time lupus assay.], beta-2 glycoprotein 1 immunoglobulins $\mathrm{M}$ and $\mathrm{G}$, anticardiolipin immunoglobulins $\mathrm{M}$ and $\mathrm{G}$, dilute Russell's viper venom time, or JAK2 V617F mutations).

Extracted data included patient age, sex, type of thrombophilia test ordered, ordering primary service, admission diagnosis, and objective confirmation of thrombotic events. The indication for test ordering was determined via medical record review of the patient's corresponding hospitalization. Each test was evaluated in the context of the patient's presenting history, hospital course, active medications, accompanying laboratory and radiographic studies, and consultant recommendations to arrive at a conclusion regarding both the test's reason for ordering and whether its indication was "inappropriate," "appropriate," or "equivocal." Cost data were obtained through the Centers for Medicare \& Medicaid Services (CMS) Clinical Laboratory Fee Schedule for 2016 (see Supplementary Material). ${ }^{6}$

The criteria for defining test appropriateness were formulated by utilizing a combination of major society guidelines and literature review. ${ }^{5,-10}$ The criteria placed emphasis upon the ordered tests' clinical relevance and reliability and were subsequently reviewed by a senior hematologist with specific expertise in thrombosis (see Supplementary Material). 
TABLE. General Testing Data Characteristics

\begin{tabular}{|c|c|c|c|}
\hline Unique Patients & Number of Tests ${ }^{a}$ & Number of Inappropriate Tests (\%) & Cost of Inappropriate Ordering (USD) \\
\hline 299 & 1817 & $777(42.7)$ & $\$ 40,422$ \\
\hline DVT & 424 & $157(37.0)$ & $\$ 8517$ \\
\hline PE & 500 & $251(50.2)$ & $\$ 13,758$ \\
\hline CVA & 458 & $158(34.5)$ & $\$ 7343$ \\
\hline Splanchnic vein thrombosis & 88 & $41(46.6)$ & $\$ 2127$ \\
\hline Other & 57 & $20(35.1)$ & $\$ 1099$ \\
\hline
\end{tabular}

a Some tests were ordered in the setting of synchronous thrombotic diagnoses, resulting in the sum of testing by site of thrombosis exceeding the total number of tests.

NOTE: Abbreviations: CVA, cerebrovascular accident; DVT, deep venous thrombosis; PE, pulmonary embolism; PVD, peripheral vascular disease; USD, US dollars.

Two internal medicine resident physician data reviewers independently evaluated the ordered tests. To ensure consistency between reviewers, a sample of identical test orders was compared for concordance, and a Cohen's kappa coefficient was calculated. For purposes of analysis, equivocal orders were included under the appropriate category, as this study focused on the quantification of potentially inappropriate ordering practices. Pearson chi-square testing was performed in order to compare ordering practices between services using Stata. ${ }^{11}$

\section{RESULTS}

In total, we reviewed 2179 individual tests, of which 362 (16.6\%) were excluded. The remaining 1817 tests involved 299 patients across 26 primary specialties. Fifty-two $(2.9 \%$ of orders) were ultimately deemed equivocal. The Table illustrates the overall proportion and cost of inappropriate test ordering as well as testing characteristics of the most commonly encountered thrombotic diagnoses. The Figure illustrates the proportion of potentially inappropriate test ordering with its associated cost by test type.

Orders for Factor V Leiden, prothrombin 20210, and protein $\mathrm{C}$ and $\mathrm{S}$ activity levels were most commonly deemed inappropriate due to the test results' failure to alter clinical management $(97.3 \%, 99.2 \%, 99.4 \%$ of their inappropriate orders, respectively). Antithrombin testing (59.4\%) was deemed inappropriate most commonly in the setting of acute thrombosis. The lupus anticoagulant $(82.8 \%)$ was inappropriately ordered most frequently in the setting of concurrent anticoagulation.

Ordering practices were then compared between nonteaching and teaching inpatient general medicine services. We observed a higher proportion of inappropriate tests ordered by the nonteaching services as compared to the teaching services (120 of 173 orders [69.4\%] versus 125 of 320 [39.1\%], respectively; $P<0.001)$.

The interreviewer kappa coefficient was $0.82(P<0.0001)$.

\section{DISCUSSION}

This retrospective analysis represents one of the largest examinations of inpatient thrombophilia testing practices to date. Our results illustrate the high prevalence and significant financial impact of potentially inappropriate thrombophilia testing conducted in the inpatient setting. The data confirm that, per our defined criteria, more than $90 \%$ of inherited thrombophilia testing was potentially inappropriate while the majority of acquired thrombophilia testing was appropriate, with the exception of the lupus anticoagulant.

Even when appropriately ordered, studies suggest that positive thrombophilia screening results fail to impact outcomes in most patients with VTE. In an effort to evaluate positive results' potential to provide a basis from which to extend the duration of anticoagulation, and therefore reduce the risk of a recurrent VTE, a case-control analysis was performed on a series of patients with a first-VTE event (Multiple Environmental and Genetic Assessment of risk factors for venous thrombosis [MEGA] study). ${ }^{3}$ In examining the odds ratio (OR) for recurrence between patients who did or did not undergo testing for Factor $\mathrm{V}$ Leiden, antithrombin, or protein $\mathrm{C}$ or $\mathrm{S}$ activity, the data failed to show an impact of testing on the risk of VTE recurrence (OR 1.2; confidence interval, 0.8-1.8). In fact, decision making has increasingly relied on patients' clinical characteristics rather than thrombophilia test results to guide anticoagulation duration after incident VTEs. A 2017 study illustrated that when using a clinical decision rule (Clinical Decision Rule Validation Study to Predict Low Recurrent Risk in Patients With Unprovoked Venous Thromboembolism [REVERSE criteria]) in patients with a first, unprovoked VTE, routine thrombophilia screening added little to determining the need for prolonged anticoagulation. ${ }^{12}$ These findings support the limited clinical utility of current test ordering practices for the prediction and management of recurrent venous thrombosis.

Regarding the acquired thrombophilias, antiphospholipid antibody testing was predominantly ordered in a justified manner, which is consistent with the notion that test results could affect clinical management, such as anticoagulation duration or choice of anticoagulant. ${ }^{13}$ However, the validity of lupus anticoagulant testing was limited by the frequency of patients on concurrent anticoagulation.

Financially, the cumulative cost associated with inappropriate ordering was substantial, regardless of the thrombotic 


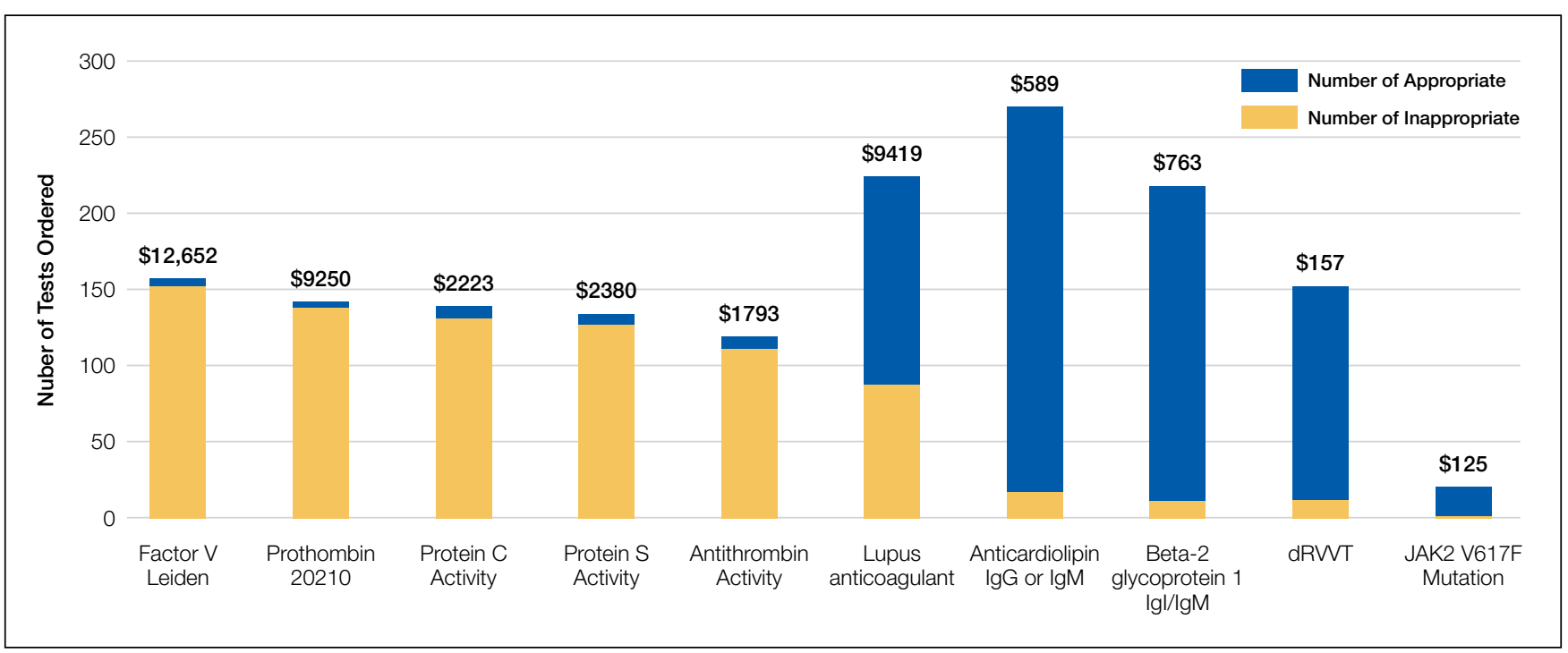

FIG. Proportion of Inappropriate Test Ordering with Associated Cost, by Test Type. Each bar represents the total number of orders and the proportion of inappropriate/appropriate orders for each thrombophilia test. The cost figures above each bar show the total cost of the corresponding test's inappropriate orders, according to the 2016 Centers for Medicare and Medicaid Services (CMS) Clinical Laboratory Fee Schedule.

event in question. Moreover, our calculated costs are derived from CMS reimbursement rates and likely underestimate the true financial impact of errant testing given that commercial laboratories frequently charge at rates several-fold higher. On a national scale, prior analyses have suggested that the annual cost of thrombophilia testing, based on typical commercial rates, ranges from $\$ 300$ million to $\$ 672$ million. ${ }^{14}$

Researchers in prior studies have similarly examined the frequency of inappropriate thrombophilia testing and methods to reduce it. Researchers in a 2014 study demonstrated initially high rates of inappropriate inherited thrombophilia testing, and then showed marked reductions in testing and cost savings across multiple specialties following the introduction of a flowchart on a preprinted order form..${ }^{15}$ Our findings provide motivation to perform similar endeavors.

The proportional difference of inappropriate ordering observed between nonteaching- and teaching-medicine services indicates a potential role for educational interventions. We recently completed a series of lectures on high-value thrombophilia ordering for residents and are actively analyzing its impact on subsequent ordering practices. We are also piloting an electronic best practice advisory for thrombophilia test ordering. Though the advisory may be overridden, providers are asked to provide justification for doing so on a voluntary basis. We plan to evaluate its effect on our findings reported in this study.

We acknowledge that our exclusion criteria resulted in the omission of testing across a spectrum of nonthrombotic clinical conditions, raising the question of selection bias. Because there are no established guidelines to determine the appropriateness of testing in these scenarios, we chose to limit the analysis of errant ordering to the context of thrombotic events. Other limitations of this study include the analysis of equivocal orders as appropriate. However, because equivocal ordering represented less than $3 \%$ of all analyzed orders, including these as inappropriate would not have significantly altered our findings.

\section{CONCLUSIONS}

A review of thrombophilia testing practices at our institution demonstrated that inappropriate testing in the inpatient setting is a frequent phenomenon associated with a significant financial impact. This effect was more pronounced in inherited versus acquired thrombophilia testing. Testing was frequently confounded and often failed to impact patients' short- or long-term clinical management, regardless of the result.

These findings serve as a strong impetus to reduce the burden of routine thrombophilia testing during hospital admissions. Our data demonstrate a need for institution-wide changes such as implementing best practice advisories, introducing ordering restrictions, and conducting educational interventions in order to reduce unnecessary expenditures and improve patient care.

Disclosure: The authors have nothing to disclose.

\section{REFERENCES}

1. Dobesh PP. Economic burden of venous thromboembolism in hospitalized patients. Pharmacotherapy. 2009;29(8):943-953.

2. Cohn DM, Vansenne F, de Borgie CA, Middeldorp S. Thrombophilia testing for prevention of recurrent venous thromboembolism. Cochrane Database Syst Rev. 2012;12:Cd007069

3. Coppens M, Reijnders JH, Middeldorp S, Doggen CJ, Rosendaal FR. Testing for inherited thrombophilia does not reduce the recurrence of venous thrombosis. J Thromb Haemost. 2008;6(9):1474-1477.

4. Somma J, Sussman, II, Rand JH. An evaluation of thrombophilia screening in an urban tertiary care medical center: A "real world" experience. Am J Clin Pathol. 2006;126(1):120-127.

5. Hicks LK, Bering $\mathrm{H}$, Carson KR, et al. The ASH Choosing Wisely ${ }^{\circledR}$ cam- 
paign: five hematologic tests and treatments to question. Blood. 2013;122(24) 3879-3883.

6. Centers for Medicare \& Medicaid Services: Clinical Laboratory Fee Schedule Files. https://www.cms.gov/Medicare/Medicare-Fee-for-Service-Payment/ ClinicalLabFeeSched/Clinical-Laboratory-Fee-Schedule-Files.html. Accessed October 2016

7. Stevens SM, Woller SC, Bauer KA, et al. Guidance for the evaluation and treatment of hereditary and acquired thrombophilia. J Thromb Thrombolysis. 2016;41(1):154-164

8. Moll S. Thrombophilia: clinical-practical aspects. J Thromb Thrombolysis. 2015;39(3):367-378

9. Kearon C, Akl EA, Ornelas J, et al. Antithrombotic therapy for vte disease: Chest guideline and expert panel report. Chest. 2016;149(2):315-352.

10. Baglin T, Gray E, Greaves M, et al. Clinical guidelines for testing for heritable thrombophilia. Br J Haematol. 2010;149(2):209-220.

11. Stata Statistical Software [computer program]. Version Release 14. College Station, TX: StataCorp LP; 2015.

12. Garcia-Horton A, Kovacs MJ, Abdulrehman J, Taylor JE, Sharma S, Lazo-Langner A. Impact of thrombophilia screening on venous thromboembolism management practices. Thromb Res.149:76-80.

13. Schulman S, Svenungsson E, Granqvist S. Anticardiolipin antibodies predict early recurrence of thromboembolism and death among patients with venous thromboembolism following anticoagulant therapy. Duration of Anticoagulation Study Group. Am J Med. 1998;104(4):332-338.

14. Petrilli CM, Heidemann L, Mack M, Durance P, Chopra V. Inpatient inherited thrombophilia testing. J Hosp Med. 2016;11(11):801-804.

15. Smith TW, Pi D, Hudoba M, Lee AY. Reducing inpatient heritable thrombophilia testing using a clinical decision-making tool. J Clin Pathol. 2014;67(4):345-349. 